\title{
Adaptive robust simultaneous stabilization controller with tuning parameters design for two dissipative Hamiltonian systems
}

\author{
ZHONG CAO, XIAORONG HOU and WENJING ZHAO
}

\begin{abstract}
This paper investigates the problem of adaptive robust simultaneous stabilization (ARSS) of two dissipative Hamiltonian systems (DHSs), and proposes a number of results on the controller parameterization design. Firstly, an adaptive $H_{\infty}$ control design approach is presented by using the dissipative Hamiltonian structural for the case that there are both external disturbances and parametric uncertainties in two DHSs. Secondly, an algorithm for solving tuning parameters of the controller is proposed using symbolic computation. The proposed controller parameterization method avoids solving Hamilton-Jacobi-Issacs (HJI) equations and the obtained controller is easier as compared to some existing ones. Finally, an illustrative example is presented to show that the ARSS controller obtained in this paper works very well.
\end{abstract}

Key words: ARSS control, DHSs, symbolic computation, controller parameterization, tuning parameters.

\section{Introduction}

In practical control systems design, the simultaneous stabilization control problem has often to be considered because of system parameters' uncertainty, controller failure modes or systems with different modes of operation. It is a more economical approach to such problem that designing a single controller to stabilize all of the systems simultaneously. So far, there are many important results for simultaneous stabilization control in linear systems [1-4]. In general, designing simultaneous stabilization controller is

Zhong Cao, the corresponding author is with School of Physics and Electronic Engineering, Guangzhou University, Guangzhou 510006, China and also with Guangdong Provincial Engineering and Technology Research Center for Mathematical Education Software, Guangzhou, 510006, China, e-mail: zhongc@gzhu.edu.cn; Xiaorong Hou is with School of Energy Science and Engineering, University of Electronic Science and Technology of China 611731, Chengdu, China, e-mail: houxr@uestc.edu.cn; Wenjing Zhao is with Lab Center, Guangzhou University, Guangzhou 510006, China, e-mail: zhaowenjing@gzhu.edu.cn

This work was supported by the National Natural Science Foundation of China No. 61374001, National High Technology Research and Development Program of China No.2015AA015408 and the scholarship from China Scholarship Council (CSC) under the Grant CSC No. 201609945011.

Received 03.08.2017. 
very difficult for a class of nonlinear systems, but it is a work worth doing for many researchers. Some nice results were proposed for the existence of simultaneous stabilization controller of a set of nonlinear systems [5]. Through using Lyapunov function method, sufficient and necessary conditions for the existence of feedback time-invariant simultaneous stabilization controllers in single-input nonlinear systems were derived [6]. In the area of robust nonlinear systems control, the simultaneous stabilization control problem is one of important research topics, which has to be studied more and more. Sufficient conditions for simultaneous stabilization control with and without $H_{\infty}$ performance were proposed for a class of state-dependent polynomial systems [7]. The simultaneous stabilization control problem for a set of port-controlled Hamiltonian systems was investigated and some results were proposed by using the Hamiltonian function method [8,9]. Ref. [10] proposed a number of results on design of parallel simultaneous stabilization controllers for a set of port-controlled Hamiltonian systems with actuator saturation. An adaptive robust parallel simultaneous stabilization of two uncertain portcontrolled Hamiltonian systems subject to input saturation had been presented [11].

As everyone knows, the control systems have two basic constraints, which are the internal stability and the external disturbance attenuation. However, it's crucial to satisfy some desired control objectives in designing a practical control system. Finding the parameterized controller is a sophisticated and efficient way for solving various control problems. Therefore, controller parameterization is a basic problem in the control theory, which has been received considerable attentions in recent decades [12-16]. Firstly, Ref. [12] proposed polynomial parameterization of stabilizing controllers. A family of nonlinear $H_{\infty}$ controller via output feedback had been proposed [13]. Yung, Ref. [14] presented a family of $H_{\infty}$ state-feedback controller for $n$-dimensional nonlinear system by extending the state-space formulas. A family of reliable nonlinear $H_{\infty}$ controller had been proposed via solving the Hamilton-Jacobi-Issacs (HJI) inequality (or equations) [15]. These controllers obtained in [12-16] are intended to solve a class of HJI inequalities (or equations), which have actually imposed a considerable difficulty. Ref. [17] studied the generalized Hamiltonian system and proposed a family of parameterized controller in $H_{\infty}$ control. An $H_{\infty}$ controller with tuning parameters for polynomial Hamiltonian systems was proposed by using symbolic computation approach [18]. The methods in [17, 18], avoided solving HJI inequalities (or equations) through applying clear physical expression and good structure of dissipative Hamiltonian systems. But the parameterized controller is intended to solve the control problem for just one system. There are fewer works for ARSS control design of two DHSs.

Therefore, it is a challenging issue to design a controller with tuning parameters to solve ARSS problem for two DHSs. In this paper, a novel and convenient method to design an ARSS controller with tuning parameters for two DHSs is proposed. We add some items containing tuning parameters into the controller, and solve the tuning parameters ranges. Within parameters ranges, the controllers insure that the DHSs are ARSS control and can optimize the robustness for all or part of DHSs.

The remainder of this paper is organized as follows. In Section 2, the problem of ARSS for DHSs is formulated. The main contribution of this paper is then given in Sec- 
tion 3, in which ARSS controller with tuning parameters and an algorithm for solving tuning parameters are provided, respectively. We present a numerical example for illustrating effectiveness and feasibility of controller in Section 4 and conclusions follow in Section 5.

\section{Problem formulation}

Consider the following two DHSs [8]:

$$
\begin{aligned}
& \sum_{1}:\left\{\begin{array}{l}
\dot{x}=\left[J_{1}\left(x, p_{1}\right)-R_{1}\left(x, p_{1}\right)\right] \frac{\partial H_{1}\left(x, p_{1}\right)}{\partial x}+g_{1}(x) u+\bar{g}_{1}(x) \omega \\
y=g_{1}{ }^{T}(x) \frac{\partial H_{1}(x)}{\partial x}
\end{array}\right. \\
& \sum_{2}:\left\{\begin{array}{l}
\dot{\xi}=\left[J_{2}\left(\xi, p_{2}\right)-R_{2}\left(\xi, p_{2}\right)\right] \frac{\partial H_{2}\left(\xi, p_{2}\right)}{\partial \xi}+g_{2}(\xi) u+\bar{g}_{2}(\xi) \omega \\
\eta=g_{2}{ }^{T}(\xi) \frac{\partial H_{2}(\xi)}{\partial \xi}
\end{array}\right.
\end{aligned}
$$

where $x, \xi \in \mathfrak{R}^{n}$ and $y, \eta \in \mathfrak{R}^{m}$ are the states vector and the outputs of the two DHSs, respectively; $p_{1}$ and $p_{2}$ are constant unknown vectors in systems, which are sufficiently small; $u \in \mathfrak{R}^{m}$ is the controller with tuning parameters; $\omega \in \mathfrak{R}^{s}$ is the disturbance; $J_{i}(x, 0)=J_{i}(x), R_{i}(x, 0)=R_{i}(x)$ and $J_{i}\left(x, p_{i}\right)=-J_{i}^{T}\left(x, p_{i}\right) \in \Re^{n \times n}$, $0 \leqslant R_{i}\left(x, p_{i}\right) \in \mathfrak{R}^{n \times n} ; g_{i}(x) \in \mathfrak{R}^{n \times m}$ and $\bar{g}_{i}(x) \in \mathfrak{R}^{n \times s}$ are sufficiently smooth functions; $H_{i}(x, 0)=H_{i}(x)$ and $H_{i}(x)$ is the Hamiltonian function which has a local minimum at the equilibrium $x_{e}^{(i)}, i=1,2, x_{e}^{(1)}=x_{0}, x_{e}^{(2)}=\xi_{0}$.

Assumption $1 H_{i}\left(x^{(i)}\right) \in C^{2}$ and the Hessian matrix Hess $\left(H_{i}\left(x_{0}^{(i)}\right)\right)>0$ for system (1) and system (2).

Given a disturbance attenuation level $\gamma>0$, choose

$$
z=\Lambda\left(g_{1}^{T}(x) \frac{\partial H_{1}(x)}{\partial x}+g_{2}^{T}(\xi) \frac{\partial H_{2}(\xi)}{\partial \xi}\right)
$$

as the penalty function, where $\Lambda \in \mathfrak{R}^{s \times m}$ is a weighting matrix with full column rank and satisfying $\lambda\left(\Lambda^{T} \Lambda\right) \leqslant 1$, where $\lambda(\cdot)$ denotes the eigenvalue of a matrix. Then, our objective of this section is described as follows:

ARSS control: Design an $L_{2}$ feedback controller $u=\alpha(x, \xi),\left(\alpha\left(x_{0}, \xi_{0}\right)=0\right)$, such that 
R1: The $L_{2}$ gain (from $\omega$ to $z$ ) of the closed-loop system is less than $\gamma$.

R2: System (1) and system (2) are simultaneously asymptotically stable when $\omega$ vanishes.

hold simultaneously.

Then, a lemma and a definition required in next section are proposed.

Lemma 1 Consider a nonlinear system [19]:

$$
\left\{\begin{array}{l}
\dot{x}=f(x)+g(x) \omega=T(x) \frac{\partial H}{\partial x}+g(x) \omega, \quad f\left(x_{0}\right)=0 \\
y=h_{1}(x) \\
z=h_{2}(x)
\end{array}\right.
$$

where $x \in \mathfrak{R}^{n}$ is the state vector, $\omega \in \mathfrak{R}^{s}$ is the disturbances, $z \in \mathfrak{R}^{q}$ is the penalty and $H(x)$ has a local minimum at the equilibrium $x_{0}$. If there exists the function $V(x) \geqslant 0$, $\left(V\left(x_{0}\right)=0\right)$ such that HJI inequality

$$
\left(\frac{\partial V}{\partial x}\right)^{T} f(x)+\frac{1}{2 \gamma^{2}}\left(\frac{\partial V}{\partial x}\right)^{T} g(x) g(x)^{T}\left(\frac{\partial V}{\partial x}\right)+\frac{1}{2} h(x)^{T} h(x) \leqslant 0
$$

holds, it's implied that the $L_{2}$ gain of the closed-loop system (2) (from $\omega$ to z) is bounded by $\gamma$, i.e.,

$$
\int_{0}^{T}\|z\|^{2} d t \leqslant \int_{0}^{T} \gamma^{2}\|\omega\|^{2} d t, \quad \forall \omega \in L_{2}[0, \mathrm{~T}]
$$

where $\gamma$ is a positive number.

Definition 1 [20] System (4) is called zero-energy-gradient (ZEG) observable with respect to $y$ if $y(t)=0$ and $\omega(t)=0, \forall t \geqslant 0$, implies $\nabla H(x(t))=0, \forall t \geqslant 0$; system (4) is called ZEG detectable with respect to $y$ if $y(t)=0$ and $\omega(t)=0, \forall t \geqslant 0$, implies $\lim _{t \rightarrow \infty} \nabla H(x(t))=0$; system (4) is called generalized ZEG observable (detectable) if $y(t)=0, z(t)=0, \omega(t)=0, \forall t \geqslant 0$, implies $\nabla H(x(t))=0, \forall t \geqslant 0$, $\left(\lim _{t \rightarrow \infty} \nabla H(x(t))=0\right)$.

\section{Main results}

In this section, firstly, an adaptive $H_{\infty}$ controller with tuning parameters for system (1) and system (2) is proposed. Then, we present an algorithm for solving tuning parameters.

Notation 1: $\nabla H_{1}=\frac{\partial H_{1}(x)}{\partial x}, \nabla H_{2}=\frac{\partial H_{2}(\xi)}{\partial \xi}, g_{1}=g_{1}(x), \bar{g}_{1}=\bar{g}_{1}(x), g_{2}=g_{2}(\xi), \bar{g}_{2}=\bar{g}_{2}(\xi)$. 


\subsection{ARSS control of two DHSs}

Assumption 2 There exits an $n_{1} \times m$ matrix $\Psi$ such that

$$
\left[J_{i}\left(x_{i}, p_{i}\right)-R_{i}\left(x_{i}, p_{i}\right)\right] \Delta_{H_{i}}\left(x_{i}, p_{i}\right)=g_{i} \Psi^{T} \theta
$$

where $\Delta_{H_{i}}\left(x_{i}, p_{i}\right)=\frac{\partial H_{i}}{\partial x_{i}}\left(x_{i}, p_{i}\right)-\frac{\partial H_{i}}{\partial x_{i}}\left(x_{i}\right)$ and $\theta \in \mathfrak{R}^{n_{1}}$ is a constant unknown parameter vector depending on $p_{i}, i=1,2, x=x_{1}, x_{2}=\xi$.

Assumption 2 is a common assumption in the adaptive control of Hamiltonian systems. In most cases, we can find and such that (7) holds [20]. For the above ARSS control problem, we have the following result.

Theorem 1 Considering system (1) and system (2), with the penalty function (3) and the given disturbance attenuation level $\gamma>0$, assume that system (1) and system (2) are generalized ZEG detectable (when $u=0$ ). If

- i) There exists a symmetric matrix $K \in \mathfrak{R}^{m \times m}$, satisfying

$$
K\left(\Lambda^{T} \Lambda+\frac{1}{\gamma^{2}} I_{m}\right)=\left(\Lambda^{T} \Lambda+\frac{1}{\gamma^{2}} I_{m}\right) K
$$

such that

$$
\left\{\begin{array}{l}
\widetilde{R}_{1}\left(x, p_{1}\right)=R_{1}\left(x, p_{1}\right)+K_{11}(x, x)-\frac{1}{2 \gamma^{2}} \bar{g}_{1} \bar{g}_{1}^{T}-\frac{1}{2} g_{1} \Lambda^{T} \Lambda g_{1}^{T} \geqslant 0 \\
\widetilde{R}_{2}\left(\xi, p_{2}\right)=R_{2}\left(\xi, p_{2}\right)-K_{22}(\xi, \xi)-\frac{1}{2 \gamma^{2}} \bar{g}_{2} \bar{g}_{2}^{T}-\frac{1}{2} g_{2} \Lambda^{T} \Lambda g_{2}^{T} \geqslant 0
\end{array}\right.
$$

where $K_{i j}(x, \xi)=\frac{1}{2} g_{i}(x) K\left(\Lambda^{T} \Lambda+\frac{1}{\gamma^{2}} I_{m}\right) g_{j}^{T}(\xi), i, j=1,2$;

- ii)

$$
g_{1} g_{2}^{T}=0 \text { and } \bar{g}_{1} \bar{g}_{2}^{T}=0
$$

- iii)

$$
\left[\nabla H_{1}^{T} g_{1}+\nabla H_{2}^{T} g_{2}\right] \Phi(x, \xi, \varphi) \leqslant 0 ;
$$

hold simultaneously. Then

$$
\left\{\begin{array}{l}
u=\frac{1}{2} K\left(\Lambda^{T} \Lambda+\frac{1}{\gamma^{2}} I_{m}\right)\left(-g_{1}^{T} \nabla H_{1}+g_{2}^{T} \nabla H_{2}\right)+\Phi(x, \xi, \varphi)-\Psi^{T} \hat{\theta} \\
\dot{\hat{\theta}}=Q \Psi\left(g_{1}^{T} \nabla H_{1}+g_{2}^{T} \nabla H_{2}\right)
\end{array}\right.
$$

is an adaptive $L_{2}$ disturbance attenuation controller, such that both $\mathbf{R} \mathbf{1}$ and $\mathbf{R 2}$ hold simultaneously for system (1) and system (2), where $\Phi(x, \xi, \varphi)$ is a polynomial matrix with a set of tuning parameters, $\varphi$ is a set of tuning parameters and $I_{m}$ is an $m \times m$ unit matrix. 
Proof Substituting controller (11) into system (1) and system (2), and Assumption 2 holds, we obtain the following closed-loop systems:

$$
\left\{\begin{array}{l}
\dot{x}=\left[J_{1}\left(x, p_{1}\right)-R_{1}\left(x, p_{1}\right)\right] \frac{\partial H_{1}\left(x, p_{1}\right)}{\partial x} \\
+g_{1}\left[\frac{1}{2} K\left(\Lambda^{T} \Lambda+\frac{1}{\gamma^{2}} I_{m}\right)\left(-g_{1}^{T} \nabla H_{1}+g_{2}^{\mathrm{T}} \nabla H_{2}\right)+\Phi(x, \xi, \varphi)\right]-g_{1} \Psi^{T}(\theta-\hat{\theta})+\bar{g}_{1} \omega \\
\dot{\xi}=\left[J_{2}\left(\xi, p_{2}\right)-R_{2}\left(\xi, p_{2}\right)\right] \frac{\partial H_{2}\left(\xi, p_{2}\right)}{\partial \xi} \\
\quad+g_{2}\left[\frac{1}{2} K\left(\Lambda^{T} \Lambda+\frac{1}{\gamma^{2}} I_{m}\right)\left(-g_{1}^{T} \nabla H_{1}+g_{2}^{\mathrm{T}} \nabla H_{2}\right)+\Phi(x, \xi, \varphi)\right]-g_{2} \Psi^{T}(\theta-\hat{\theta})+\bar{g}_{2} \omega \\
\dot{\hat{\theta}}=Q \Psi\left(g_{1}^{T} \nabla H_{1}+g_{2}^{\mathrm{T}} \nabla H_{2}\right)
\end{array}\right.
$$

The system (12) can rewrite as:

$$
\begin{aligned}
& {\left[\begin{array}{c}
\dot{x} \\
\dot{\xi} \\
\dot{\hat{\theta}}
\end{array}\right]=\left[\begin{array}{ccc}
J_{1}\left(x, p_{1}\right)-R_{1}\left(x, p_{1}\right)-K_{11}(x, x) & K_{12}(x, \xi) & -g_{1} \Psi^{T} Q \\
-K_{21}(\xi, x) & J_{2}\left(\xi, p_{2}\right)-R_{2}\left(\xi, p_{2}\right)+K_{22}(\xi, \xi) & -g_{2} \Psi^{T} Q \\
\left(g_{1} \Psi^{T} Q\right)^{T} & \left(g_{2} \Psi^{T} Q\right)^{T} & 0
\end{array}\right]} \\
& \times\left[\begin{array}{c}
\frac{\partial H_{1}\left(x, p_{1}\right)}{\partial x} \\
\frac{\partial H_{2}\left(\xi, p_{2}\right)}{\partial x} \\
-Q^{-1}(\theta-\hat{\theta})
\end{array}\right]+\left[\begin{array}{c}
g_{1}(x) \\
g_{2}(\xi) \\
0
\end{array}\right] \Phi(x, \xi, \hat{\theta})+\left[\begin{array}{c}
\bar{g}_{1}(x) \\
\bar{g}_{2}(\xi) \\
0
\end{array}\right] \omega
\end{aligned}
$$

Let $X=\left[x^{T}, \xi^{T}, \hat{\theta}^{T}\right]^{T}, Y=\left[y^{T}, \eta^{T}\right]^{T}$,

$$
\begin{aligned}
& \bar{J}(X, p)=\left[\begin{array}{ccc}
J_{1}\left(x, p_{1}\right) & K_{12}(x, \xi) & -g_{1} \Psi^{T} Q \\
-K_{21}(\xi, x) & J_{2}\left(\xi, p_{2}\right) & -g_{2} \Psi^{T} Q \\
\left(g_{1} \Psi^{T} Q\right)^{T} & \left(g_{2} \Psi^{T} Q\right)^{T} & 0
\end{array}\right], \\
& \bar{R}(X, p)=\left[\begin{array}{ccc}
R_{1}\left(x, p_{1}\right)+K_{11}(x, x) & 0 & 0 \\
0 & R_{2}\left(\xi, p_{2}\right)-K_{22}(\xi, \xi) & 0 \\
0 & 0 & 0
\end{array}\right], G(X)=\left[\begin{array}{c}
g_{1}(x) \\
g_{2}(\xi) \\
0
\end{array}\right],
\end{aligned}
$$$$
\bar{G}(X)=\left[\begin{array}{c}
\bar{g}_{1}(x) \\
\bar{g}_{2}(\xi) \\
0
\end{array}\right], \bar{H}(X)=H_{1}(x)+H_{2}(\xi)+\frac{1}{2}(\theta-\hat{\theta})^{T} Q^{-1}(\theta-\hat{\theta}) \text {, where }
$$

$$
\frac{\partial \bar{H}(X)}{\partial X}=\left[\begin{array}{c}
\frac{\partial \bar{H}(X)}{\partial x} \\
\frac{\partial \bar{H}(X)}{\partial \xi} \\
\frac{\partial \bar{H}(X)}{\partial \hat{\theta}}
\end{array}\right]=\left[\begin{array}{c}
\nabla H_{1} \\
\nabla H_{2} \\
\nabla H_{3}
\end{array}\right], \quad p=\left[p_{1}{ }^{T}, p_{2}{ }^{T}\right]^{T}, \quad v=\Phi(x, \xi, \varphi) .
$$


So, the systems (13) can be rewritten as:

$$
\left\{\begin{array}{l}
\dot{X}=[\bar{J}(X, p)-\bar{R}(X, p)] \frac{\partial \bar{H}(X)}{\partial X}+G(X) v+\bar{G}(X) \omega=f(X)+\bar{G}(X) \omega \\
Y=M(X) \frac{\partial \bar{H}(X)}{\partial X} .
\end{array}\right.
$$

Obviously, $\bar{J}(X, p)$ is a skew-symmetric matrix and $\bar{R}(X, p)$ is a positive semi-definite matrix. Thus, the system (14) is a dissipative Hamiltonian system (DHS).

The output of the system (1) and system (2) can be rewritten as:

$$
Y=M(X) \frac{\partial \bar{H}(X)}{\partial X}
$$

where $M(X)=\left[\begin{array}{ccc}g_{1}^{T}(x) & 0 & 0 \\ 0 & g_{2}^{T}(\xi) & 0 \\ 0 & 0 & 0\end{array}\right]$

The penalty function can be expressed as:

$$
z=\Lambda G^{T}(X) \frac{\partial \bar{H}(X)}{\partial X}:=h(X) .
$$

Consider the candidate Lyapunov function $V(X)=\bar{H}(X)-c \geqslant 0$, where $c=\bar{H}\left(X_{0}\right)$. With Lemma 1 and the conditions of the Theorem 1, we have

$$
\begin{aligned}
& \left(\frac{\partial V}{\partial X}\right)^{T} f(X)+\frac{1}{2 \gamma^{2}}\left(\frac{\partial V}{\partial X}\right)^{T} \bar{G}(X) \bar{G}^{T}(X)\left(\frac{\partial V}{\partial X}\right)+\frac{1}{2} h^{T}(X) h(X) \\
& =-\left(\frac{\partial \bar{H}}{\partial X}\right)^{T} \bar{R}(X, p) \frac{\partial \bar{H}}{\partial X}+\left(\frac{\partial \bar{H}}{\partial X}\right)^{T} G(X) v+\frac{1}{2 \gamma^{2}}\left(\frac{\partial \bar{H}}{\partial X}\right)^{T} \bar{G}(X) \bar{G}^{T}(X)\left(\frac{\partial \bar{H}}{\partial X}\right) \\
& +\frac{1}{2} h^{T}(X) h(X) \\
& =-\left[\begin{array}{lll}
\nabla H_{1}^{T} & \nabla H_{2}^{T} & \nabla H_{3}^{T}
\end{array}\right]\left[\begin{array}{ccc}
R_{1}\left(x, p_{1}\right)+K_{11}(x, x) & R_{2}\left(\xi, p_{2}\right)-K_{22}(\xi, \xi) & 0 \\
0 & 0 & 0
\end{array}\right]\left[\begin{array}{c}
\nabla H_{1} \\
\nabla H_{2} \\
\nabla H_{3}
\end{array}\right] \\
& \left.+\left[\begin{array}{lll}
\nabla H_{1}^{T} & \nabla H_{2}^{T} & \nabla H_{3}^{T}
\end{array}\right]\left[\begin{array}{c}
g_{1} \\
g_{2} \\
0
\end{array}\right] \begin{array}{c}
0 \\
\end{array}\right]
\end{aligned}
$$




$$
\begin{aligned}
& +\frac{1}{2 \gamma^{2}}\left[\begin{array}{lll}
\nabla H_{1}^{T} & \nabla H_{2}^{T} & \nabla H_{3}^{T}
\end{array}\right]\left[\begin{array}{c}
\bar{g}_{1} \\
\bar{g}_{2} \\
0
\end{array}\right]\left[\begin{array}{lll}
\bar{g}_{1}^{T} & \bar{g}_{2}^{T} & 0
\end{array}\right]\left[\begin{array}{c}
\nabla H_{1} \\
\nabla H_{2} \\
\nabla H_{3}
\end{array}\right] \\
& +\frac{1}{2}\left[\begin{array}{lll}
\nabla H_{1}^{T} & \nabla H_{2}^{T} & \nabla H_{3}^{T}
\end{array}\right]\left[\begin{array}{c}
g_{1} \\
g_{2} \\
0
\end{array}\right] \Lambda^{T} \Lambda\left[\begin{array}{lll}
g_{1}^{T} & g_{2}^{T} & 0
\end{array}\right]\left[\begin{array}{c}
\nabla H_{1} \\
\nabla H_{2} \\
\nabla H_{3}
\end{array}\right] \\
& =-\left(\nabla H_{1}^{T}\left(R_{1}+K_{11}\right) \nabla H_{1}+\nabla H_{2}^{T}\left(R_{2}-K_{22}\right) \nabla H_{2}\right)+\left(\nabla H_{1}^{T} g_{1}+\nabla H_{2}^{T} g_{2}\right) \Phi(x, \xi, \varphi) \\
& +\frac{1}{2 \gamma^{2}}\left(\nabla H_{1}^{T} \bar{g}_{1} \bar{g}_{1}^{T} \nabla H_{1}+\nabla H_{2}^{T} \bar{g}_{2} \bar{g}_{1}^{T} \nabla H_{1}+\nabla H_{1}^{T} \bar{g}_{1} \bar{g}_{2}^{T} \nabla H_{2}+\nabla H_{2}^{T} \bar{g}_{2} \bar{g}_{2}^{T} \nabla H_{2}\right) \\
& +\frac{1}{2}\left(\nabla H_{1}^{T} g_{1} \Lambda^{T} \Lambda g_{1}^{T} \nabla H_{1}+\nabla H_{2}^{T} g_{2} \Lambda^{T} \Lambda g_{1}^{T} \nabla H_{1}\right. \\
& \left.+\nabla H_{1}^{T} g_{1} \Lambda^{T} \Lambda g_{2}^{T} \nabla H_{2}+\nabla H_{2}^{T} g_{2} \Lambda^{T} \Lambda g_{2}^{T} \nabla H_{2}\right) \\
& =-\nabla H_{1}^{T}\left(R_{1}+K_{11}-\frac{1}{2 \gamma^{2}} \bar{g}_{1} \bar{g}_{1}^{T}-\frac{1}{2} g_{1} \Lambda^{T} \Lambda g_{1}^{T}\right) \nabla H_{1} \\
& -\nabla H_{2}^{T}\left(R_{2}-K_{22}-\frac{1}{2 \gamma^{2}} \bar{g}_{2} \bar{g}_{2}^{T}-\frac{1}{2} g_{2} \Lambda^{T} \Lambda g_{2}^{T}\right) \nabla H_{2} \\
& +\left(\nabla H_{1}^{T} g_{1}+\nabla H_{2}^{T} g_{2}\right) \Phi(x, \xi, \varphi) \leqslant 0
\end{aligned}
$$

According to Lemma 1, the $L_{2}$ gain of system (14) (from $\omega$ to $z$ ) is no more than $\gamma$ and R1 holds.

Next, we prove that system (14) is asymptotically stable when $\omega=0$. When $\omega=0$, it is easy to know from system (14) that

$$
\begin{aligned}
& \dot{V}(X)=\left(\frac{\partial V}{\partial X}\right)^{T}[\bar{J}(X, p)-\bar{R}(X, p)]\left(\frac{\partial V}{\partial X}\right)+\left(\frac{\partial V}{\partial X}\right)^{T} G(X) v \\
& =-\left(\frac{\partial \bar{H}(X)}{\partial X}\right)^{T} \bar{R}(X, p)\left(\frac{\partial \bar{H}(X)}{\partial X}\right)+\left(\frac{\partial \bar{H}(X)}{\partial X}\right)^{T} G(X) v \\
& =-\left[\begin{array}{lll}
\nabla H_{1}^{T} & \nabla H_{2}^{T} & \nabla H_{3}^{T}
\end{array}\right]\left[\begin{array}{ccc}
R_{1}\left(x, p_{1}\right)+K_{11}(x, x) & R_{2}\left(\xi, p_{2}\right)-K_{22}(\xi, \xi) & 0 \\
0 & 0 & 0
\end{array}\right]\left[\begin{array}{c}
\nabla H_{1} \\
\nabla H_{2} \\
\nabla H_{3}
\end{array}\right] \\
& +\left[\begin{array}{lll}
\nabla H_{1}^{T} & \nabla H_{2}^{T} & \nabla H_{3}^{T}
\end{array}\right]\left[\begin{array}{c}
g_{1} \\
g_{2} \\
0
\end{array}\right] \Phi(x, \xi, \varphi)
\end{aligned}
$$




$$
\begin{aligned}
= & -\nabla H_{1}^{T}\left(R_{1}+K_{11}-\frac{1}{2 \gamma^{2}} \bar{g}_{1} \bar{g}_{1}^{T}-\frac{1}{2} g_{1} \Lambda^{T} \Lambda g_{1}^{T}\right) \nabla H_{1}-\frac{1}{2 \gamma^{2}} \nabla H_{1}^{T} \bar{g}_{1} \bar{g}_{1}^{T} \nabla H_{1} \\
& -\frac{1}{2} \nabla H_{1}^{T} g_{1} \Lambda^{T} \Lambda g_{1}^{T} \nabla H_{1}-\nabla H_{2}^{T}\left(R_{2}-K_{22}-\frac{1}{2 \gamma^{2}} \bar{g}_{2} \bar{g}_{2}^{T}-\frac{1}{2} g_{2} \Lambda^{T} \Lambda g_{2}^{T}\right) \nabla H_{2} \\
& -\frac{1}{2 \gamma^{2}} \nabla H_{2}^{T} \bar{g}_{2} \bar{g}_{2}^{T} \nabla H_{2}-\frac{1}{2} \nabla H_{2}^{T} g_{2} \Lambda^{T} \Lambda g_{2}^{T} \nabla H_{2} \\
& +\left(\nabla H_{1}^{T} g_{1}+\nabla H_{2}^{T} g_{2}\right) \Phi(x, \xi, \varphi) \leqslant 0
\end{aligned}
$$

Thus, the solution of the closed-loop system converges to the largest invariant set contained in

$$
S=\{X: \dot{V}(X)=0\} \subset\left\{X: Y=M(X) \nabla \bar{H} \equiv 0, z=\Lambda G^{T}(X) \nabla \bar{H} \equiv 0, \forall t \geqslant 0\right\}
$$

From the fact that system (14) is generalized ZEG detectable, we know that

$$
\lim _{t \rightarrow \infty} \nabla \bar{H}(X(t))=\lim _{t \rightarrow \infty}\left(\begin{array}{c}
\nabla H(x(t)) \\
\nabla H(\xi(t)) \\
-Q^{-1}(\theta-\hat{\theta}(t))
\end{array}\right)=\left(\begin{array}{c}
\nabla H\left(\lim _{t \rightarrow \infty} x(t)\right) \\
\nabla H\left(\lim _{t \rightarrow \infty} \xi(t)\right) \\
\lim _{t \rightarrow \infty}\left[-Q^{-1}(\theta-\hat{\theta}(t))\right]
\end{array}\right)=0
$$

$$
\forall X \in S
$$

Hence, the largest invariant set contains only one point, i.e., $X_{0}=\left[x_{0}^{T}, \xi_{0}^{T}, \hat{\theta}^{T}\right]^{T}$ which is the equilibrium point. From LaSalle's invariance principle, the closed-loop system (14) and controller (11) is asymptotically stable at its equilibrium and R2 holds. This completes the proof.

\section{Remark 1}

1. In Theorem, when all of tuning parameters $\varphi$ in controller (11) are zero (i.e., $\Phi(x, \xi, \varphi)=0)$, the controller is same to the controller proposed [7], which is a controller without parameter. However, the controller (11) obtained in this paper is a parameterized controller, which has a set of tuning parameters.

2. The condition (8) and (9) in Theorem are not restrictive, and can be easily satisfied in many systems.

3. $\Phi(x, \xi, \varphi)$ is a polynomial vector with tuning parameters. We can obtain the tuning parameters range of via solving condition (10).

4. The proposed parameterization method can be used for a nonlinear control system, and of course the first step in applying the method is to express the nonlinear system as a DHS based on dissipative Hamiltonian realization methods [21, 22]. 


\subsection{Solving of tuning parameters (STP) algorithm}

From condition (8), we can obtain the $\gamma^{*}$. Let $\gamma \geqslant \gamma^{*}$ such that condition (8) holds. Then we propose an algorithm to find tuning parameters ranges of controller (11) via solving tuning parameters of $\Phi(x, \xi, \varphi)$ in condition (10). The STP algorithm now proceeds as follows.

\section{STP Algorithm}

INPUT: A set parameters of system $S y s=\left\{x_{i_{N}}, N_{1}, N_{2}, r, \nabla H_{1}, \nabla H_{2}, G_{1}, G_{2}\right\} . / / x_{i_{N}}$ is the state variable of two systems, $N_{1}$ is the number of the state variable of system one, $N_{2}$ is the number of state variable of system two and $r$ is the highest order polynomial for tuning parameters.

OUTPUT: A set of Tuning parameters' ranges $U . / / U$ is empty set initially.

Step 1: Initialization

For $i=1$ to $l \quad / / l=N_{1}$

For $k=1$ to $r$

$$
\begin{aligned}
& \left\{t=c(l+k-1, k) ; k=k_{1}+k_{2}+\cdots+k_{l} ; P_{i l}=\sum_{j=1}^{t} v_{j l} x_{i 1}^{k_{1}} x_{i 2}^{k_{2}} \cdots x_{i l}^{k_{l}} ;\right. \\
& \left.\Phi_{a}=\Phi_{a}+P_{i l} ;\right\} \\
& / / c(l+k-1, k) \text { is the combinatorial function. }
\end{aligned}
$$

For $i=1$ to $l \quad / / l=N_{2}$

For $k=1$ to $r$

$$
\begin{gathered}
\left\{t=c(l+k-1, k) ; k=k_{1}+k_{2}+\cdots+k_{l} ; P_{i l}=\sum_{j=1}^{t} v_{j l} x_{i 1}^{k_{1}} x_{i 2}^{k_{2}} \cdots x_{i l}^{k_{l}} ;\right. \\
\left.\Phi_{b}=\Phi_{b}+P_{i l} ;\right\} \\
\Phi\left(X_{1}, X_{2}, v\right)=\left[\Phi_{a}, \Phi_{b}\right]^{T}
\end{gathered}
$$


Step 2: Main body

Begin

$S=-\left[\nabla H_{1}^{T} G_{1}+\nabla H_{2}^{T} G_{2}\right] \Phi\left(X_{1}, X_{2}, v\right)$

For $j=1$ to nops $(S) ; \quad / / \operatorname{nops}(S)$ is the number of terms of polynomial $S$.

If $(\operatorname{deg}(S)$ is odd number); $\quad / / \operatorname{deg}(S)$ is degree of polynomial $S$.

$\Theta_{1}=\{\operatorname{coef}(S, \operatorname{deg}(S)=$ odd $)=0\} ; \quad / /$ Choose some terms, which degree is odd number $(\operatorname{deg}(S)=$ odd $)$ from $S$ and let the coefficients of such terms be zero. We can obtain a set of equations $\Theta_{1}$.

\section{End If}

$U_{1}=\left\{\right.$ CylindricalAlgebraicDecompose $\left.\left(\Theta_{1}\right)\right\} ; \quad$ //Solve the $\Theta_{1}$ by using cylindrical algebraic decompositions (CAD) algorithm [23] and obtain the solution set $U_{1}$ of the tuning parameters.

$S^{\prime}=\operatorname{subs}\left(U_{1}, S\right) ; \quad / /$ Substitute solution set $U_{1}$ into $S$ and obtain a new polynomial $S^{\prime}$. $M=\operatorname{Matrix}\left(S^{\prime}\right) ; \quad / /$ Rewrite $S^{\prime}$ as coefficient matrix $M$.

$\Theta_{2}=\{$ Determinant $(M) \geqslant 0\} ; \quad / /$ All principal minors of $M$ must be positive semi-definite [24] and we can obtain a set of inequalities $\Theta_{2}$.

$U_{2}=\left\{\right.$ CylindricalAlgebraicDecompose $\left.\left(\Theta_{2}\right)\right\} ; \quad / /$ Solve the $\Theta_{2}$ by using CAD and choose one solution set of tuning parameters ranges $U_{2}$.

$U=U_{1} \cup U_{2}$ and Return $(U)$;

End. This completes the algorithm. 


\section{Remark 2}

1. The STP algorithm starts from $r=1$ normally.

2. The CAD algorithm is given in Semi-Algebraic-Set-Tools of Regular-Chains in Maple 16.

3. It is merely to simplify computation that we let some tuning parameters be zero before using $\mathrm{CAD}$ algorithm. However, these tuning parameters are not necessarily zero. The solution set of tuning parameters obtained by STP algorithm is a subset of solutions.

\section{Numerical experiments}

Consider the following two DHSs with external disturbances and parametric uncertainties described as:

$$
\left\{\begin{array}{l}
\dot{x}=\left[J_{1}\left(x, p_{1}\right)-R_{1}\left(x, p_{1}\right)\right] \frac{\partial H_{1}\left(x, p_{1}\right)}{\partial x}+g_{1}(x) u+\bar{g}_{1}(x) \omega \\
y=g_{1}{ }^{T}(x) \frac{\partial H_{1}(x)}{\partial x}
\end{array}\right.
$$

where

$$
\begin{aligned}
J_{1}\left(x, p_{1}\right)=\left[\begin{array}{ccc}
0 & 1 & 0 \\
-1 & 0 & -2 \\
0 & 2 & 0
\end{array}\right], g_{1}=\left[\begin{array}{cc}
0 & 0 \\
1 & -1 \\
1 & -1
\end{array}\right], \quad \bar{g}_{1}=\left[\begin{array}{ll}
0 & 0 \\
1 & 1 \\
0 & 0
\end{array}\right] \\
R_{1}\left(x, p_{1}\right)=\operatorname{Diag}\left\{1+p_{1}, 1,2\right\}, H_{1}\left(x, p_{1}\right)=\frac{1}{2} x_{1}^{2}+x_{2}^{2}+\frac{1}{2}\left(1+p_{1}\right) x_{3}^{2}, \quad\left|p_{1}\right|<1 . \\
\qquad \begin{array}{l}
\dot{\xi}=\left[J_{2}\left(\xi, p_{2}\right)-R_{2}\left(\xi, p_{2}\right)\right] \frac{\partial H_{2}\left(\xi, p_{2}\right)}{\partial \xi}+g_{2}(\xi) u+\bar{g}_{2}(\xi) \omega \\
\eta=\bar{g}_{2}^{T}(\xi) \frac{\partial H_{2}(\xi)}{\partial \xi}
\end{array}
\end{aligned}
$$

where

$$
\begin{gathered}
J_{2}\left(\xi, p_{2}\right)=\left[\begin{array}{ccc}
0 & -1 & 0 \\
1 & 0 & 0 \\
0 & 0 & 0
\end{array}\right], g_{2}=\left[\begin{array}{cc}
1 & 1 \\
-1 & -1 \\
0 & 0
\end{array}\right], \bar{g}_{2}=\left[\begin{array}{cc}
1 & -1 \\
-1 & 1 \\
0 & 0
\end{array}\right] \\
R_{2}\left(\xi, p_{2}\right)=\operatorname{Diag}\left\{1,2,1+p_{2}\right\}, H_{2}\left(\xi, p_{2}\right)=\left(1+p_{2}\right) \xi_{1}{ }^{2}+2 \xi_{2}{ }^{2}+\xi_{3}{ }^{2}, \quad\left|p_{2}\right|<1
\end{gathered}
$$




\subsection{Controller design and solving tuning parameters}

From system (20) and system (21), it is easy to get

$\operatorname{Hess}\left(H_{1}\left(x_{0}\right)\right)=\left[\begin{array}{ccc}1 & 0 & 0 \\ 0 & 2 & 0 \\ 0 & 0 & 1+p_{1}\end{array}\right]>0, \quad \operatorname{Hess}\left(H_{2}\left(\xi_{0}\right)\right)=\left[\begin{array}{ccc}2+2 p_{2} & 0 & 0 \\ 0 & 4 & 0 \\ 0 & 0 & 2\end{array}\right]>0$.

So Assumption 1 holds.

Given a disturbance attenuation level $\gamma>0$, choose

$$
z=\Lambda\left(g_{1}^{T} \nabla H_{1}+g_{2}^{T} \nabla H_{2}\right) \text {. }
$$

Suppose $K=\left[\begin{array}{cc}1 & 0 \\ 0 & -1\end{array}\right], \Lambda=\left[\begin{array}{cc}\frac{1}{2} & 0 \\ 0 & \frac{1}{2}\end{array}\right]$. We have $K^{T} K=\left[\begin{array}{cc}1 & 0 \\ 0 & 1\end{array}\right], \Lambda^{T} \Lambda=$ $\left[\begin{array}{cc}\frac{1}{4} & 0 \\ 0 & \frac{1}{4}\end{array}\right], K\left(\Lambda^{T} \Lambda+\frac{1}{\gamma^{2}} I_{m}\right)=\left(\Lambda^{T} \Lambda+\frac{1}{\gamma^{2}} I_{m}\right) K=\left[\begin{array}{cc}\frac{1}{4}+\frac{1}{\gamma^{2}} & 0 \\ 0 & -\frac{1}{4}-\frac{1}{\gamma^{2}}\end{array}\right]$. A straightforward computation shows that when $\gamma \geqslant \frac{2 \sqrt{15}}{5}$,

$$
\widetilde{R}_{1}(x)=\left[\begin{array}{ccc}
1+p_{1} & 0 & 0 \\
0 & \frac{3}{4}-\frac{1}{\gamma^{2}} & -\frac{1}{4} \\
0 & -\frac{1}{4} & \frac{7}{4}
\end{array}\right] \geqslant 0, \quad \widetilde{R}_{2}(\xi)=\left[\begin{array}{ccc}
\frac{3}{4}-\frac{1}{\gamma^{2}} & \frac{1}{4}+\frac{1}{\gamma^{2}} & 0 \\
\frac{1}{4}+\frac{1}{\gamma^{2}} & \frac{7}{4}-\frac{1}{\gamma^{2}} & 0 \\
0 & 0 & 1+p_{2}
\end{array}\right] \geqslant 0 .
$$

Thus, the condition (8) holds. From system (20) and system (21),

$$
g_{1} g_{2}^{T}=\left[\begin{array}{lll}
0 & 0 & 0 \\
0 & 0 & 0 \\
0 & 0 & 0
\end{array}\right], \quad \bar{g}_{1} \bar{g}_{2}^{T}=\left[\begin{array}{ccc}
0 & 0 & 0 \\
0 & 0 & 0 \\
0 & 0 & 0
\end{array}\right]
$$

The condition (9) holds.

Let $\Phi(x, \xi, \varphi)=\left[\begin{array}{l}\Phi_{1}\left(x, \varphi_{1}\right) \\ \Phi_{2}\left(\xi, \varphi_{2}\right)\end{array}\right]$. We know that $n=6$ in system (20) and system (21). Let $r=1$, We have $\Phi_{1}\left(x, \varphi_{1}\right)=a_{1} x_{1}+a_{2} x_{2}+a_{3} x_{3}, \Phi_{2}\left(\xi, \varphi_{2}\right)=b_{1} \xi_{1}+b_{2} \xi_{2}+b_{3} \xi_{3}$, where $\varphi_{1}$ is the tuning parameters $a_{i}$ and $\varphi_{2}$ is the tuning parameters $b_{i}, i=1,2,3$.

From system (20) and system (21), we obtain that

$$
\left[\nabla H_{1}^{T} g_{1}+\nabla H_{2}^{T} g_{2}\right]=\left[\begin{array}{ll}
2 x_{2}+x_{3}+2 \xi_{1}-4 \xi_{2} & -2 x_{2}-x_{3}+2 \xi_{1}-4 \xi_{2}
\end{array}\right] .
$$

Let $S=-\left[\nabla H_{1}^{T} g_{1}+\nabla H_{2}^{T} g_{2}\right] \Phi(x, \xi)$, we have

$$
\begin{gathered}
S=-2 a_{1} x_{1} x_{2}-a_{1} x_{1} x_{3}-2 a_{1} x_{1} \xi_{1}+4 a_{1} x_{1} \xi_{2}-2 a_{2} x_{2}^{2}-\left(a_{2}+2 a_{3}\right) x_{2} x_{3}-\left(2 a_{2}-2 b_{1}\right) x_{2} \xi_{1} \\
+\left(4 a_{2}+2 b_{2}\right) x_{2} \xi_{2}+2 b_{3} x_{2} \xi_{3}-a_{3} x_{3}^{2}-\left(2 a_{3}-b_{1}\right) x_{3} \xi_{1}+\left(4 a_{3}+b_{2}\right) x_{3} \xi_{2}+b_{3} x_{3} \xi_{3} \\
-2 b_{1} \xi_{1}^{2}+\left(4 b_{1}-2 b_{2}\right) \xi_{1} \xi_{2}-2 b_{3} \xi_{1} \xi_{3}+4 b_{2} \xi_{2}^{2}+4 b_{3} \xi_{2} \xi_{3} .
\end{gathered}
$$


$S$ is a quadratic form and can be rewritten as a coefficient matrix (multiply constant 2 for simplified calculation).

$$
M=\left[\begin{array}{cccccc}
0 & -2 a_{1} & -a_{1} & -2 a_{1} & 4 a_{1} & 0 \\
-2 a_{1} & -4 a_{2} & -2 a_{3}-a_{2} & -2 a_{2}+2 b_{1} & 4 a_{2}+2 b_{2} & 2 b_{3} \\
-a_{1} & -2 a_{3}-a_{2} & -2 a_{3} & -2 a_{3}+b_{1} & 4 a_{3}+b_{2} & b_{3} \\
-2 a_{1} & -2 a_{2}+2 b_{1} & -2 a_{3}+b_{1} & -4 b_{1} & 4 b_{1}-2 b_{2} & -2 b_{3} \\
4 a_{1} & 4 a_{2}+2 b_{2} & 4 a_{3}+b_{2} & 4 b_{1}-2 b_{2} & 8 b_{2} & 4 b_{3} \\
0 & 2 b_{3} & b_{3} & -2 b_{3} & 4 b_{3} & 0
\end{array}\right]
$$

All the principal minors of $M$ must be positive semi-definite. We have inequalities $B$ from $M$. From $B$, we can easily obtain that $a_{3} \leqslant 0, b_{1} \leqslant 0$.

Substituting $U_{1}=\left\{a_{1}=0, b_{3}=0, a_{2}=2 a_{3}, b_{2}=-2 b_{1}\right\}$ into inequalities $B$ for simplify computation, we obtain simplified inequalities $B^{\prime}$. Solving inequalities $B^{\prime}$ by using CAD algorithm, we obtain a series of sets. Choose some sets, which satisfy inequalities $B^{\prime}$, and organize them. We have

$$
\left[U=\left\{a_{3} \leqslant 0, b_{1} \leqslant 0,12 a_{3} b_{1}-4 a_{3}^{2}-b_{1}^{2} \geqslant 0\right\} \cup U_{1} .\right.
$$

From system (20) and system (21), we have

$$
\begin{aligned}
& {\left[-g_{1}^{T} \nabla H_{1}+g_{2}^{T} \nabla H_{2}\right]=\left[\begin{array}{ll}
-2 x_{2}-x_{3}+2 \xi_{1}-4 \xi_{2} & 2 x_{2}+x_{3}+2 \xi_{1}-4 \xi_{2}
\end{array}\right]^{T},} \\
& {\left[g_{1}^{T} \nabla H_{1}+g_{2}^{T} \nabla H_{2}\right]=\left[\begin{array}{ll}
2 x_{2}+x_{3}+2 \xi_{1}-4 \xi_{2} & -2 x_{2}-x_{3}+2 \xi_{1}-4 \xi_{2}
\end{array}\right]^{T} .}
\end{aligned}
$$

Substituting them and $U$ into controller (11), we obtain the controller:

$$
\left\{\begin{array}{l}
u=\left[\begin{array}{c}
\left(\frac{1}{8}+\frac{1}{2 \gamma^{2}}\right)\left(-2 x_{2}-x_{3}+2 \xi_{1}-4 \xi_{2}\right) \\
\left(-\frac{1}{8}-\frac{1}{2 \gamma^{2}}\right)\left(2 x_{2}+x_{3}+2 \xi_{1}-4 \xi_{2}\right)
\end{array}\right]+\left[\begin{array}{c}
2 a_{3} x_{1}+a_{3} x_{3} \\
b_{1} \xi_{1}-2 b_{1} \xi_{2}
\end{array}\right]-\Psi^{T} \hat{\theta} \\
\dot{\hat{\theta}}=Q \Psi\left[\begin{array}{c}
2 x_{2}+x_{3}+2 \xi_{1}-4 \xi_{2} \\
-2 x_{2}-x_{3}+2 \xi_{1}-4 \xi_{2}
\end{array}\right]
\end{array}\right.
$$

where $a_{3} \leqslant 0, b_{1} \leqslant 0,12 a_{3} b_{1}-4 a_{3}^{2}-b_{1}^{2} \geqslant 0$.

We have the controller with tuning parameters for system (20) and system (21). The controller (24) has a rather simple form.

\subsection{Simulations and results}

In order to evaluate the robustness of the controller (24), we set the disturbance attenuation level of system (20) and system (21) as: $\gamma=\sqrt{3}$, and the tuning parameters 
of controller as: $a_{3}=-1, b_{1}=-1$. Choose

$$
\Psi=\left[\begin{array}{ccc}
-\xi_{1} & 0 & -x_{3} \\
-\xi_{1} & 0 & x_{3}
\end{array}\right]^{T}, \quad \theta=\left[\begin{array}{lll}
p_{2} & 0 & p_{1}
\end{array}\right]^{T},
$$

we have

$$
\begin{aligned}
& {\left[J_{1}\left(x, p_{1}\right)-R_{1}\left(x, p_{1}\right)\right] \Delta_{H}\left(x, p_{1}\right)=g_{1} \Psi^{T} \theta=\left[\begin{array}{lll}
0 & -2 p_{1} x_{3} & -2 p_{1} x_{3}
\end{array}\right]^{T},} \\
& {\left[J_{2}\left(\xi, p_{2}\right)-R_{2}\left(\xi, p_{2}\right)\right] \Delta_{H}\left(\xi, p_{2}\right)=g_{2} \Psi^{T} \theta=\left[\begin{array}{lll}
-2 p_{2} \xi_{1} & 2 p_{2} \xi_{1} & 0
\end{array}\right]^{T} .}
\end{aligned}
$$

The Assumption 2 holds for system (20) and system (21). Suppose $Q=\operatorname{Diag}\{1,1,1\}$ and we have the controller (25), which has a rather simple form.

$$
\left\{\begin{array}{l}
u_{1}=-\frac{31}{12} x_{2}-\frac{31}{24} x_{3}+\frac{7}{12} \xi_{1}-\frac{7}{6} \xi_{2}+\xi_{1} \hat{\theta}_{1}+x_{3} \hat{\theta}_{3} \\
u_{2}=-\frac{7}{12} x_{2}-\frac{7}{24} x_{3}-\frac{19}{12} \xi_{1}+\frac{19}{6} \xi_{2}+\xi_{1} \hat{\theta}_{1}-x_{3} \hat{\theta}_{3} \\
\dot{\hat{\theta}}_{1}=-4 \xi_{1}^{2}+8 \xi_{1} \xi_{2} \\
\dot{\hat{\theta}}_{3}=-4 x_{2} x_{3}-2 x_{3}^{2}
\end{array}\right.
$$

To illustrate the effectiveness of controller (25), we carry out some numerical simulations with the following choices: $x(0)=[0.6,0.2,1]^{T}, \xi(0)=[0.5,1,0.2]^{T}$. To test the robustness of the controller with respect to external disturbances, a square disturbance $\omega=[2,3]^{T}$ is added to systems in the time duration $[2 \sim 2.5 s]$. The parameter perturbation $p=[0.5,0.5]^{T}$. The simulation results are shown in Figures $1-4$, which are the responses of the state and control signal, respectively.

From Figures 1-4, we know that the controller (25) is very effective in ARSS control for system (20) and system (21).

In order to evaluate the robustness optimization of the systems by adjusting the tuning parameters of controller (24), we choose the parameters of system (20) and system (21) as: $a_{3}=-1, b_{1}=-100$. We have the following controller:

$$
\left\{\begin{array}{l}
u_{1}=-\frac{31}{12} x_{2}-\frac{31}{24} x_{3}+\frac{7}{12} \xi_{1}-\frac{7}{6} \xi_{2}+\xi_{1} \hat{\theta}_{1}+x_{3} \hat{\theta}_{3} \\
u_{2}=-\frac{7}{12} x_{2}-\frac{7}{24} x_{3}-\frac{1207}{12} \xi_{1}+\frac{1207}{6} \xi_{2}+\xi_{1} \hat{\theta}_{1}-x_{3} \hat{\theta}_{3} \\
\dot{\hat{\theta}}_{1}=-4 \xi_{1}^{2}+8 \xi_{1} \xi_{2} \\
\dot{\hat{\theta}}_{3}=-4 x_{2} x_{3}-2 x_{3}^{2}
\end{array}\right.
$$

To illustrate the effectiveness of controller (26), the initial value of systems and parameter perturbation are same to above. We impose an external disturbance $\omega=[2,3]^{T}$ on system (20) and system (21) during the time period $22.5 \mathrm{~s}$. 


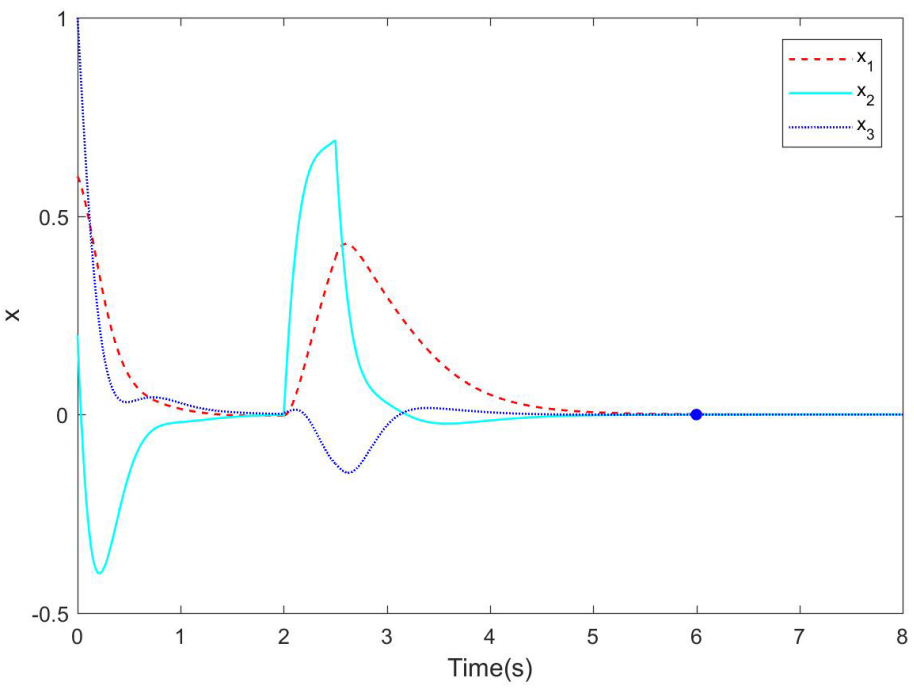

Figure 1: Swing curves of $x$

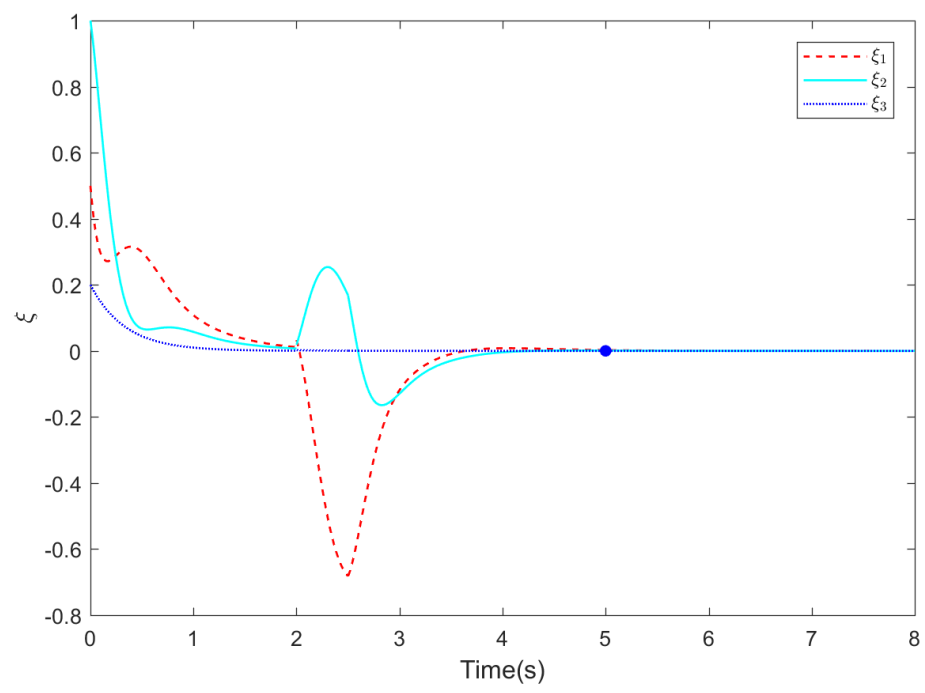

Figure 2: Swing curves of $\xi$

The simulation results are shown in Figure 5 and Figure 6, which are the response of the state of system (20) and system (21) under controller (26), respectively.

From Figure 5 and Figure 6, we know that the controller (26) is very effective in ARSS control for system (20) and system (21). From Figure 5, we can clearly see that 


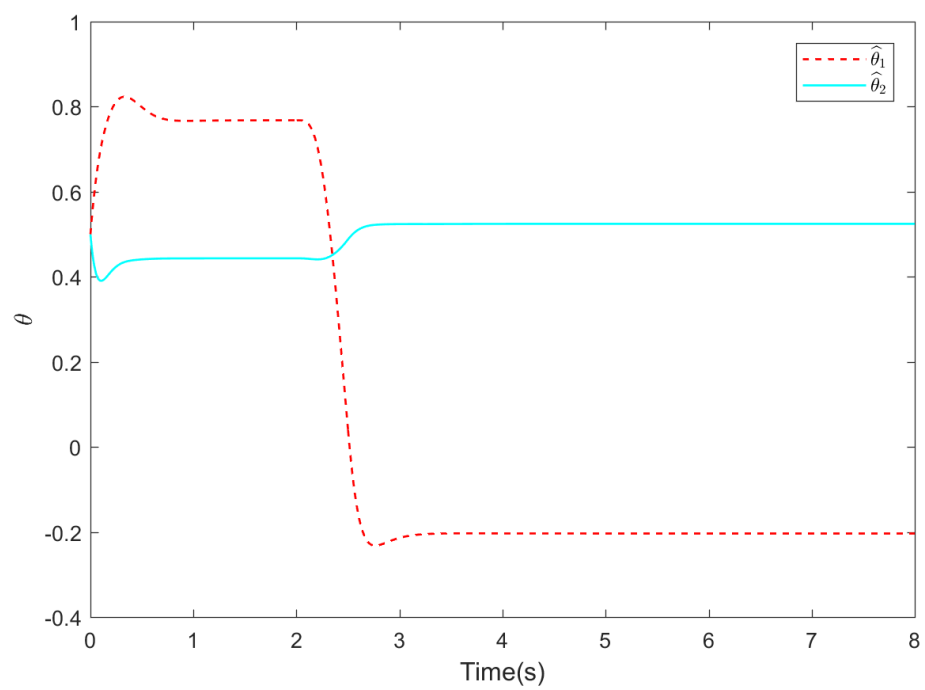

Figure 3: Swing curves of $\hat{\theta}$

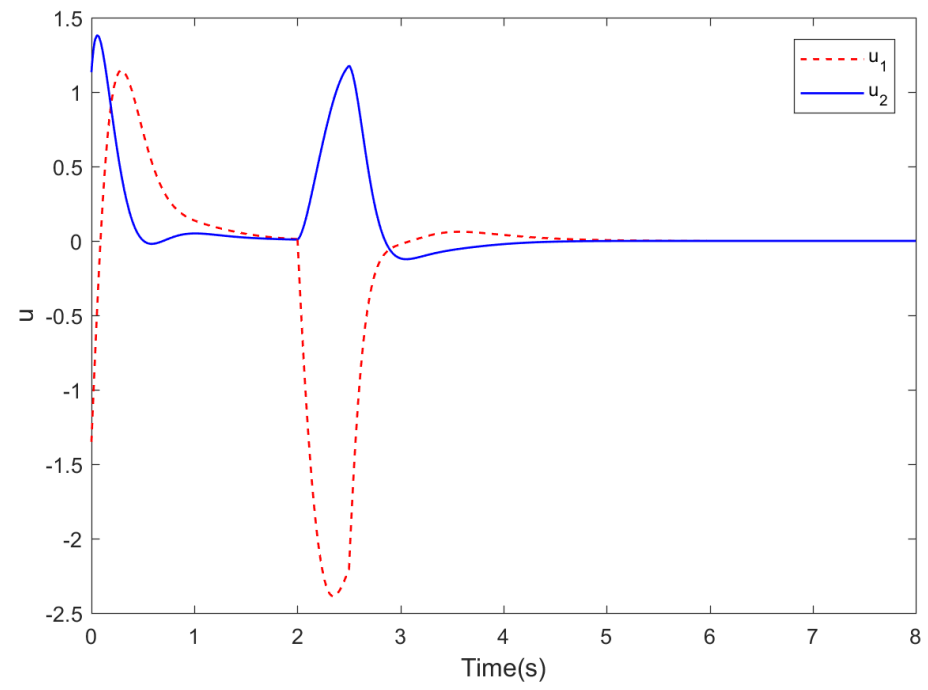

Figure 4: Swing curves of $u$

under controller (26), it takes 5.5 seconds for the system to back to the equilibrium point (circle point in Figures), while under controller (25), it takes about 6.0 seconds in Figure 1. The controller (26) is a little better in robust performance for system (20) than controller (25). However, from Figure 6, we can clearly see that under controller 


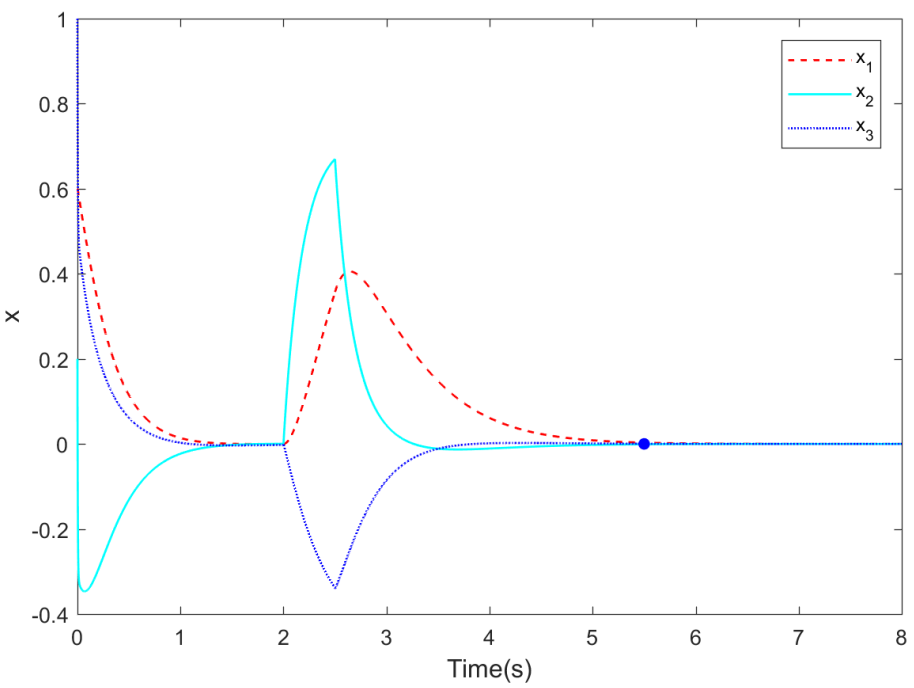

Figure 5: Swing curves of $x$

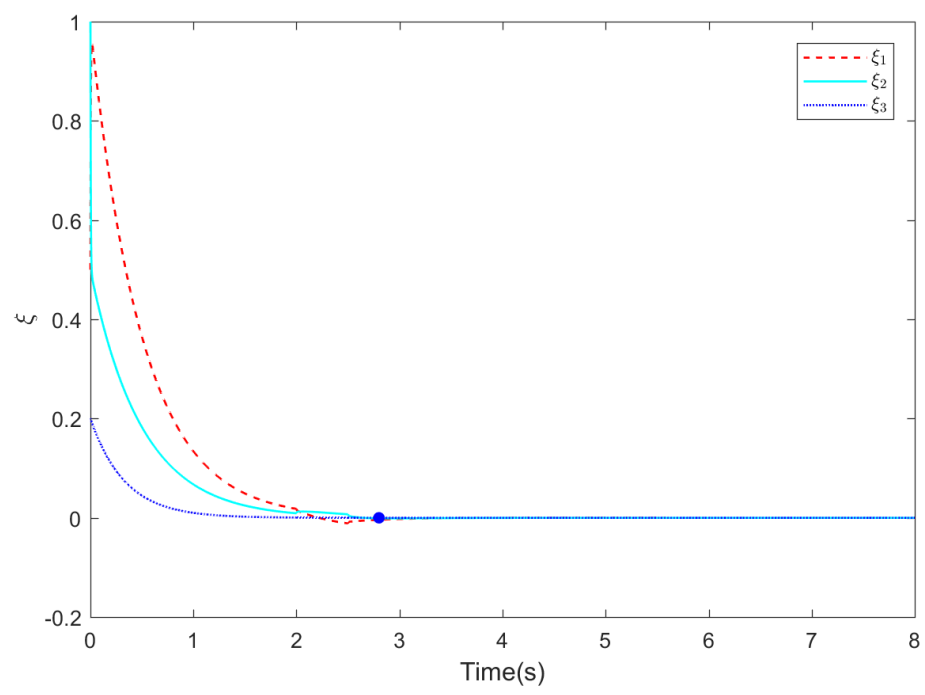

Figure 6: Swing curves of $\xi$

(26), it takes 2.8 seconds for the system to back to the equilibrium point, while under controller (25), it takes about 5.0 seconds in Figure 2. The time of state $\xi$ back to the equilibrium point is much shorter in Figure 6 than in Figure 2. With the adjustment control parameters, the system achieved a more robust effect. 
Simulation shows that the controller with tuning parameters is effective as it can optimize $H_{\infty}$ control by adjusting the tuning parameters values. Within the tuning parameters ranges, the controllers insure that the dissipative systems are adaptive robust simultaneous stabilization control and can optimize the robustness for all or part of Hamiltonian systems and insure that the others complex control objectives by adjusting the tuning parameters values.

\section{Conclusions}

In this paper, we have investigated the ARSS problem for two DHSs, and proposed an $H_{\infty}$ ARSS controller with tuning parameters design method. A controller with tuning parameters has been obtained using Hamiltonian function method and an algorithm for solving tuning parameters of the controller has been proposed with symbolic computation. The proposed controller parameterization method avoids solving HJI equations and the obtained controller is easier as compared to some existing ones. The numerical experiment and simulations show that the ARSS controller is strongly robust in disturbances and unknown parameters, and meanwhile the ARSS controller can optimize the robustness of the system by adjusting tuning parameters.

\section{References}

[1] G.D. HowITT and R. LuUs: Simultaneous stabilization of linear single-input systems by linear state feedback control. Int. J. of Control, 54(4), (1991), 1015-1030.

[2] M. Paskota, V. Sreeram, K.L. Teo and A.I. Mees: Optimal simultaneous stabilization of linear single-input systems via linear state feedback control. Int. J. of Control, 60(4), (1994), 483-498.

[3] D. MiLleR and D Kennedy: Simultaneous stabilization with near-optimal model reference tracking. Systems \& Control Letters, 46(1), (2002), 31-43.

[4] X. Wang, A. Saberi, A.A. Stoorvogel and P. Sannuti: Simultaneous global external and internal stabilization of linear time-invariant discrete-time systems subject to actuator saturation. Automatica, 48(5), (2012), 699-711.

[5] B. Ho-Mock-QAI and W.P. DaYaWANSA: Simultaneous stabilization of linear and nonlinear systems by means of nonlinear state feedback. SIAM J. on Control and Optimization, 37(6), (1999), 1701-1725.

[6] J.L. WU: Simultaneous stabilization for a collection of single-input nonlinear systems. IEEE Trans. on Automatic Control, 50(3), (2005), 328-337. 
[7] J. XU, L. XIE and Y. WANG: Simultaneous stabilization and robust control of polynomial nonlinear systems using SOS techniques. IEEE Trans. on Automatic Control, 54(8), (2009), 1892-1897.

[8] Y. WANG, G. FENG and D. Cheng: Simultaneous stabilization of a set of nonlinear port-controlled Hamiltonian systems. Automatica, 43(3), (2007), 403-415.

[9] L. SUN and Y. WANG: Simultaneous stabilization of a class of nonlinear descriptor systems via Hamiltonian function method. Science in China Series F: Information Sciences, 52(11), (2009), 2140-2152.

[10] A. WEI, Y. WANG and X. Hu: Parallel simultaneous stabilization of a set of portcontrolled Hamiltonian systems subject to actuator saturation. J. of Systems Science and Complexity, 24(1), (2011), 120-139.

[11] A. WEI, Y. WANG and X. HU: Adaptive robust parallel simultaneous stabilization of two uncertain port-controlled Hamiltonian systems subject to input saturation. The 31st Chinese Control Conf. (CCC), (2012), 727-732.

[12] D.C. Youla, H. JABR and J.J. Bongiorno JR: Modern Wiener-Hopf design of optimal controllers, Part II: The multivariable case. IEEE Trans. on Automatic Control, 21 (1976), 319-338.

[13] W.M. LU and J.C. DOYLE: Control of nonlinear systems via output feedback: controller parameterization. IEEE Trans. on Automatic Control, 39(12), (1994), 2517-2521.

[14] C.F. Yung, J.L. Wu and T.T. LEE: Parameterization of nonlinear $H_{\infty}$ statefeedback controllers. Automatica, 33(8), (1997), 1587-1590.

[15] Y.S. Fu, Z.H TiAn, and S.J. SHI: A family of reliable $H_{\infty}$ state-feedback controllers for nonlinear systems with strictly redundant actuators: the full information case. J. of Systems Science and Systems Engineering, 10(3), (2001), 350-358.

[16] J. Gadewadikar, F. L. Lewis, L. Xie, V. Kucera and M. Abu-Khalaf: Parameterization of all stabilizing $H_{\infty}$ static state-feedback gains: Application to output-feedback design. Automatica, 43(9), (2007), 1597-1604.

[17] S. XU and X. HoU: A family of $H_{\infty}$ controllers for dissipative Hamiltonian systems. Int. J. of Robust and Nonlinear Control, 22(11), (2012), 1258-1269.

[18] Z. CAO and X. HoU: A Symbolic computation approach to parameterizing controller for polynomial Hamiltonian systems. Mathematical Problems in Engineering, Article ID 806428, (2014).

[19] T. SHEN: $H_{\infty}$ Control Theory and its Applications. Tsinghua University Press, Beijing, 1996. 
[20] A. VAN DER SChAFT: $L_{2}$-Gain and Passivity Techniques in Nonlinear Control. Springer, Berlin, 2012.

[21] Y. WAng, D. Chen, C. LI and Y. Ge: Dissipative Hamiltonian realization and energy-based $L_{2}$-disturbance attenuation control of multimachine power systems. IEEE Trans. on Automatic Control, 48(8), (2003), 1428-1433.

[22] Y. WANG, D. Chen and S. GE: Approximate dissipative Hamiltonian realization and construction of local Lyapunov function. Systems \& Control Letters, 56(2), (2007), 141-149.

[23] B.F. CAVINESS and J.R. JOHNSON: Quantifier elimination and cylindrical algebraic decomposition. Springer Science \& Business Media, 1998.

[24] K.N. Swamy: On Sylvesters criterion for positive-semidefinite matrices. IEEE Trans. on Automatic Control, 18(3), (1973), 306. 